\title{
Processing speed impairment in schizophrenia is mediated by white matter integrity
}

\author{
Haleh Karbasforoushan, Brittney Duffy, Jennifer Urbano Blackford, and Neil D. Woodward \\ Psychotic Disorders \& Psychiatric Neuroimaging Programs, Department of Psychiatry, Vanderbilt \\ University School of Medicine, Nashville, TN
}

\begin{abstract}
Background-Processing speed predicts functional outcome and is a potential endophenotype for schizophrenia. Establishing the neural basis of processing speed impairment may inform the treatment and etiology of schizophrenia. Neuroimaging investigations in healthy subjects have linked processing speed to brain anatomical connectivity. However, the relationship between processing speed impairment and white matter integrity in schizophrenia is unclear.
\end{abstract}

Methods-Individuals with schizophrenia and healthy subjects underwent diffusion tensor imaging (DTI) and completed a brief neuropsychological assessment that included measures of processing speed, verbal learning, working memory, and executive functioning. Group differences in white matter integrity, inferred from fractional anisotropy (FA), were examined throughout the brain and the hypothesis that processing speed impairment in schizophrenia is mediated by diminished white matter integrity was tested.

Results-White matter integrity of the corpus callosum, cingulum, superior and inferior frontal gyri, and precuneus was reduced in schizophrenia. Average FA in these regions mediated group differences in processing speed, but not other cognitive domains. Diminished white matter integrity in schizophrenia was accounted for, in large part, by individual differences in processing speed.

Conclusions-Cognitive impairment in schizophrenia mediated by reduced white matter integrity. This relationship was strongest for processing speed as deficits in working memory, verbal learning, and executive functioning were not mediated by white integrity. Larger sample sizes may be required to detect more subtle mediation effects in these domains. Interventions that preserve white matter integrity or ameliorate white matter disruption may enhance processing speed and functional outcome in schizophrenia.

\section{Introduction}

Schizophrenia is characterized by widespread neuropsychological impairment (Dickinson et al. 2008; Heinrichs and Zakzanis, 1998). Among the specific domains affected, processing

Address Correspondence to: Neil D. Woodward, Ph.D., Jack Martin, M.D., Research Professor in Psychopharmacology, Psychiatric Neuroimaging \& Psychotic Disorders Programs, Vanderbilt Psychiatric Hospital, Suite 3057, $160123^{\text {rd }}$ Ave. S., Nashville, TN 37212 , Phone: 615.322.8361, Fax: 615.936.3563.

Disclosures: No commercial support was received for the preparation of this manuscript and the authors have no conflicts of interest to report. 
speed is the most impaired with patients performing approximately 1.5 SDs below healthy comparison subjects on average (Dickinson et al. 2007). Processing speed impairment is an important predictor of functional outcome in patients, may predict conversion to a fullblown psychotic disorder in at-risk subjects, and is also present in unaffected relatives of probands (Dickinson et al. 2007; Fusar-Poli et al. 2012; Nuechterlein et al. 2011; Sanchez et al. 2009). Consequently, elucidating the neural basis of processing speed impairment has the potential to improve our understanding of the etiology and treatment of schizophrenia.

Given the nature of neuropsychological tests of processing speed, which require subjects to rapidly perform relatively simple tasks that integrate a number of basic cognitive operations, it is not surprising that neuroimaging investigations of the neural basis of processing speed have focused on anatomical brain connectivity. In healthy subjects, processing speed is associated with white matter integrity, inferred from fractional anisotropy quantified with diffusion tensor imaging (DTI), in a number of brain areas, including prefrontal white matter, longitudinal fasciculus, external capsule, optic radiation, cingulum, and corpus callosum (Kerchner et al. 2012; Koch et al. 2012; Salami et al. 2012; Sasson et al. 2013; Tuch et al. 2005; Turken et al. 2008). Some of these associations appear to be specific to processing speed. For instance, white matter integrity in the cingulum, corpus callosum, and corona radiata has been reliably linked to processing speed, whereas correlations between white matter integrity and executive functions and memory are often limited to frontal and temporal lobe regions, respectively (Bendlin et al. 2010; Sasson et al. 2013). However, there is also evidence that white matter integrity throughout the brain is related to processing speed and, more broadly, intellectual functioning (Penke et al. 2010; Penke et al. 2012).

Evidence of widespread reductions in white matter integrity in schizophrenia provides a possible neural basis for processing speed impairment (Fitzsimmons et al. 2013; Melonakos et al. 2011; Yao et al. 2013). A wide array of white matter regions/tracts have been implicated in schizophrenia, including the corpus callosum, cingulum, and longitudinal fasciculus. However, the findings are remarkably heterogeneous raising the possibility that white matter disruption in schizophrenia may vary as a function of individual differences in cognitive impairment. Emerging evidence suggests that this is indeed the case as the integrity of a number of white matter tracts, including the cingulum, uncinate fasciculus, longitudinal fasciculi, internal capsule, and thalamocortical connections has been linked to several cognitive abilities, including processing speed, working memory, executive functioning, and verbal memory (Karlsgodt et al. 2008; Levitt et al. 2012; Marenco et al. 2012; Nestor et al. 2004; Nestor et al. 2010; Perez-Iglesias et al. 2010; Qiu et al. 2009; Szeszko et al. 2007; Takei et al. 2009; Voineskos et al. 2012). Nonetheless, several questions remain unaddressed. First, the extent to which cognitive impairment, especially slowed processing speed, is mediated by white matter integrity remains unclear. Second, the specificity of cognition-white matter associations has not been established. Finally, it is not known if disrupted white matter integrity in schizophrenia is entirely attributable to cognitive impairment. That is, is there a categorical "disease" effect on white matter integrity, or does variation along the dimension of cognition account for altered white matter integrity in schizophrenia? We explored these questions using tract-based spatial statistics (TBSS) analysis of DTI data and neuropsychological testing. In light of strong evidence that processing speed in particular is related to white matter integrity in healthy subjects, we 
tested the hypothesis that processing speed impairment in schizophrenia is mediated by diminished white matter integrity and that this relationship is would be strongest for processing speed compared to other cognitive domains. We further explored the extent to which cognitive impairment accounts for reduced white matter integrity in schizophrenia.

\section{Methods}

\section{Participants and Study Procedures}

26 schizophrenia/schizoaffective patients and 20 healthy subjects were recruited to participate in this study, which was approved by the Vanderbilt University Institutional Review Board. All subjects provided written informed consent prior to participation. Individuals with schizophrenia were recruited from the Psychotic Disorders Program at the Vanderbilt Psychiatric Hospital in Nashville, TN. Healthy subjects were recruited from Nashville and surrounding area via print/internet advertisement and word-of-mouth. All study participants were administered the Structured Clinical Interview for Diagnosing DSMIV Disorders (SCID: First et al. 1996) to confirm diagnoses in patients and rule out current or past psychiatric illness in healthy subjects. Exclusion criteria included: age less than 18 or greater than 60; estimated pre-morbid intellect less than 70; presence of a systemic medical illness or neurological disorder that would affect study results; reported pregnancy or lactation; history of significant head trauma; psychotropic drug use (healthy controls only); substance abuse within the past three months (patients); lifetime history of substance abuse/ dependence (healthy controls); and MRI contra-indicators (e.g. metal implants, claustrophobia).

\section{Neuropsychological Testing}

Subjects completed the Wechsler Test of Adult Reading (WTAR: Wechsler, 2001), a singleword reading test of estimated pre-morbid intellectual functioning, and the Screen for Cognitive Impairment in Psychiatry (SCIP: Purdon, 2005). The SCIP is a brief cognitive battery that includes 5 sub-tests: 1 ) a word-list learning test of verbal memory comprised of 3 learning trials of a 10-word list; 2) a working memory test modeled after the Auditory Consonant Trigram Test; 3) phonemic verbal fluency test of executive functioning; 4) a visuomotor coding test of processing speed modeled after the Coding subtest from the Wechsler Adult Intelligence Scales (Wechsler, 1997); and 5) a delayed recall trial of the word list. Each sub-test was administered and scored according to published guidelines.

\section{Neuroimaging Data Acquisition and Preprocessing}

Diffusion weighted images (DWIs) and T1-weighted anatomical MR image were collected from each subject on a research dedicated 3T Philips Intera Achieva scanner (Philips Healthcare, Best, the Netherlands). Diffusion weighted images were acquired using an eightchannel sensitivity encoding (SENSE) head coil with the following parameters: TR $=10,000$ $\mathrm{ms} ; \mathrm{TE}=48 \mathrm{~ms} ; \mathrm{FOV}=240 \times 240 \mathrm{~mm}^{2} ; \mathrm{B}_{0}$ image and 92 diffusion directions at $\mathrm{B}=1000$ $\mathrm{s} / \mathrm{mm}^{2}$; flip angle $=90^{\circ} ;$ matrix size $=96 \times 96$; voxel size $=2.5 \mathrm{~mm}$ isotropic; number of slices $=52$. The high resolution T1-weighted anatomical image included a 170 sagital slices with $1.0 \mathrm{~mm}$ isotropic resolution. The DWIs were pre-processed using the FMRIB Software Library version 4.1.4 (FSL: Oxford Centre for Functional MRI of the Brain, UK; http:// 
www.fmrib.ox.ac.uk/fsl/) (Behrens et al. 2003; Smith et al. 2004). Raw DTI data were corrected for eddy current distortions using the Eddy Current Correction (ECC) tool and for motion using the McFlirt Motion Correction tool. The FMRIB Brain Extraction Tool (BET; Smith, 2002) was then used for skull stripping. Diffusion tensors were fitted at each voxel and fractional anisotropy (FA) images were created using the DTIFIT tool of FMRIB FDT toolbox-version 2.0 (Behrens et al. 2003).

\section{Tract-Based Spatial Statistics Analysis}

Voxel-wise statistical analysis of the DTI FA images was carried out using Tract-Based Spatial Statistics (TBSS; Smith et al. 2006) in FSL. First, each subjects' FA image was aligned to the standard $1 \times 1 \times 1 \mathrm{~mm}$ MNI152 template in FSL using the non-linear registration tool (FNIRT). Next, the mean FA image was created and thinned to create a mean FA skeleton representing the centers of all tracts using a threshold of 0.25 . Each subject's aligned FA map was then projected onto this skeleton resulting in each subject's skeletonised FA image.

\section{Statistical Analyses}

Given our a-priori interest in understating the neural correlates of processing speed impairment, a mediation analysis was performed to estimate the direct and indirect relationships between group, white matter integrity, and processing speed. Parameter estimates were computed for each path in the mediation analysis: A, B, C, C' (see Figure 1B). First, the association between neuropsychological functioning and diagnostic group (i.e. path C) was established by carrying out an independent groups t-test. Once this was confirmed, we tested to see if there was a relationship between group and the mediator variable, white matter integrity (i.e. path A). Group differences in voxel-wise FA were examined by entering each subjects' skeletonised FA into a general linear model (twosample t-test) design matrix with non-parametric permutation testing using the Randomize tool in FSL (10,000 permutations). The results were thresholded at $\mathrm{p}=.05$ (corrected) using the threshold-free cluster enhancement (TFCE) option to find clusters without setting an initial cluster level (Smith and Nichols, 2009). From this, we extracted the mean FA from all voxels demonstrating a group difference from each subjects' skeletonized FA image. Thus, each subject contributed a single value to the mediation analysis that reflected mean FA within the regions identified in the between groups analysis. Mean FA was then regressed onto processing speed (i.e. path B). Finally, a regression analysis with group and the mediator variable (i.e. mean FA from regions showing between groups differences) entered as predictors was performed (i.e. path $\mathrm{C}^{\prime}$ ). We used the Sobel test to determine the significance of the mediation effect. We also used a bootstrap method (Preacher and Hayes, 2004) to confirm that results of the Sobel test based on evidence that traditional methods have normality assumptions that are rarely met (Hayes, 2009) or suffer from low statistical power (MacKinnon et al. 2002). Bootstrap methods are distribution-free and provide greater statistical power. We estimated the $95 \%$ confidence interval of the coefficient from the Sobel test; intervals that did not include 0 were considered statistically significant. To determine if the relationship was most robust for processing speed, we repeated the mediation analysis with the verbal learning, delayed recall, working memory, and verbal fluency sub-test scores entered as the dependent variables. 
In addition to determining if processing speed impairment in schizophrenia was mediated by white matter integrity, we also investigated the extent to which abnormal white matter integrity in schizophrenia was accounted for by cognitive impairment. That is, we were interested in determining if white matter differences associated with the categorical disease dimension were accounted for by individual differences in the dimension of cognitive impairment. To address this question, we repeated the voxel-wise between groups analysis of FA entering each of the SCIP domains, processing speed, verbal learning, verbal fluency, working memory, and delayed recall separately as covariates in ANCOVA analyses. The results from these analyses show group differences on a voxel-wise basis after covarying for impairment in each neuropsychological domain.

\section{Results}

\section{Demographics and Neuropsychological Test Scores}

Two subjects ( 1 individual with schizophrenia and 1 healthy subject) were excluded from the study due to suspected artifacts in the imaging data. Therefore, the final sample consisted of 25 schizophrenia patients and 19 healthy controls. Demographic data for the two groups is presented in Table 1. Level of education was lower in schizophrenia compared to healthy subjects $(\mathrm{t}=3.52, \mathrm{p}=.001)$. The two groups were otherwise well-matched on age, parental education, sex, race, and estimated pre-morbid intellectual functioning. With respect to clinical variables, the average age of onset and duration of illness was approximately age 20 and 19 years, respectively. Most patients were taking one atypical antipsychotic $(n=15)$, with the remaining patients taking two atypical antipsychotics $(\mathrm{n}=2)$ and one typical antipsychotic $(n=2) .3$ patients were unmedicated and medication status could not be determined for 3 patients. Average antipsychotic dosage among the medicated patients, calculated in chlorpromazine equivalents using the guidelines established by Gardner et al. (, 2010), was $515.3 \pm 216.5 \mathrm{mg} /$ day. Consistent with expectations, schizophrenia patients performed worse on all subtests of the SCIP, except the verbal fluency subtest (see Table 1).

\section{Group Differences in White Matter Integrity}

Whole brain voxel-wise analysis revealed decreased FA in a number of brain regions in schizophrenia, including the genu, body and splenium of the corpus callosum, cingulum, bilateral superior and inferior frontal sub-gyral white matter, bilateral anterior corona radiata, and right precuneus white matter (see Figure 1A). There were no significant increases in FA in schizophrenia patients compared to healthy controls.

\section{Relationship Between Cognitive Impairment in Schizophrenia and White Matter Integrity}

Consistent with our hypothesis, processing speed impairment in schizophrenia was partially mediated by white matter integrity (Figure 1B). Specifically, patients performed worse than healthy subjects on the SCIP processing speed subtest (i.e. path C: $\mathrm{t}(42)=4.85, \mathrm{p}<.0001$ ) and this effect was substantially attenuated when white matter integrity was added as an additional predictor (i.e. path $\mathrm{C}^{\prime}: \mathrm{t}(42)=3.15, \mathrm{p}=.003$ ). Sobel test confirmed that the relationship between schizophrenia and processing speed was partially mediated by white matter integrity $(\beta=.48$, bootstrap Confidence Interval $=.18-.92, \mathrm{p}<.05)$. Repetition of the mediation analysis with verbal learning, working memory, verbal fluency, and delayed recall 
entered as the dependent variable confirmed that the relationship was strongest for processing speed (see Supplemental Table 1). Specifically, despite the fact that verbal learning, working memory, and delayed recall were robustly impaired in schizophrenia; FA did not mediate these relationships ( $\beta$ values ranged from .11-.31 and all confidence intervals for the mediation test included 0 ). To further investigate the selectivity of the relationship between white matter and processing speed, we calculated a SCIP composite score by converting raw sub-test scores to Z-scores using published normative data and averaging them together, excluding the processing speed measure, and performed a mediation analysis with SCIP composite score as the dependent variable. As expected, the SCIP composite score was lower in schizophrenia compared to healthy subjects (path C: $\mathrm{t}(42)=3.91, \mathrm{p}<.001$; effect size $=-1.20$ ). However, the effect was not substantially attenuated when white matter integrity was added as a predictor (i.e. path $\mathrm{C}^{\prime}: \mathrm{t}(42)=2.98, \mathrm{p}=.005$; Sobel test $\beta=.04$, bootstrap Confidence Interval=-.13 - .19) indicating that impaired overall cognitive ability in schizophrenia was not mediated by white matter integrity.

A voxel-wise correlation analysis was performed to further investigate the relationship between processing speed impairment and white matter integrity. Specifically, each schizophrenia patient's skeletonized FA map was entered into a general linear model with processing speed test scores entered as a predictor. As with the between groups analysis, 10,000 iterations of a random permutation method (randomise tool, FSL) and the TFCE option was used to find clusters without setting an initial cluster level. The resultant statistical maps were masked by the between group differences image, and thresholded at $\mathrm{p}$ $<0.05$. This analysis was repeated entering the remaining SCIP sub-test scores as a predictor to determine if other cognitive functions also correlated with FA. As shown in Figure 2A, several of the regions that demonstrated reduced FA in schizophrenia also positively correlated with processing speed in the patient group, including the genu and body of corpus callosum, bilateral superior and inferior frontal sub-gyral white matter, and left anterior corona radiata. In contrast, FA did not correlate with verbal learning, working memory, verbal fluency, and delayed recall (see Figure 2B-E).

\section{Group Differences in White Matter Integrity after Controlling for Cognitive Impairment}

To further examine the impact of cognitive functioning on white matter integrity in schizophrenia, the whole-brain analysis of group differences in white matter integrity was repeated included each of the SCIP subtests as a covariate in separate ANCOVA analyses. As shown in Figure 3, after accounting for processing speed, there were no significant group differences in FA anywhere in the brain, even when a more liberal threshold of $\mathrm{p}=.10$ (corrected) was used. In contrast, significant differences persisted after correcting for working memory and verbal fluency. The group differences were also mitigated to some extent when verbal learning was included as a covariate; however, the same general pattern of reduced white matter integrity observed in the no covariate analysis was apparent at the more liberal threshold of $\mathrm{p}=.10$ (corrected). 


\section{Discussion}

Cognitive impairment, especially slowed processing speed, is an important predictor of functional outcome in schizophrenia and a potential endophenotype of the disorder (Dickinson et al. 2007; Nuechterlein et al. 2011; Sanchez et al. 2009). Prompted by compelling evidence that brain anatomical connectivity is related to processing speed in healthy subjects, we investigated the relationship between white matter integrity and processing speed, and cognitive functioning more broadly, in schizophrenia. We hypothesized that: 1) consistent with prior studies, the integrity of key white matter tracts would be reduced in schizophrenia; and 2) cognitive impairment, especially processing speed impairment, would be mediated by white matter integrity. Our results support these hypotheses. Specifically, white matter integrity in the corpus callosum, frontal lobe, cingulum, anterior corona radiata, and precuneus was reduced in schizophrenia. Importantly, processing speed impairment in schizophrenia was partially mediated by white matter integrity in these regions. The relationship between cognitive functioning and white matter integrity was strongest for processing speed as impairments in other cognitive domains, including working memory, verbal learning, and executive functioning were not mediated by white matter integrity. Finally, in an effort to further understand the relationship between cognition and white matter integrity in schizophrenia, we examined the extent to which white matter reductions associated with the categorical diagnosis of schizophrenia was accounted for by variation in the dimension of cognitive impairment. The results of this analysis revealed that disrupted white matter integrity in schizophrenia is largely accounted for by individual differences in processing speed impairment. In contrast, group differences in white matter integrity persisted after impairment in other cognitive domains were accounted for, including working memory and executive functioning. Combined, our findings implicate reduced white matter integrity in the expression of cognitive impairment in schizophrenia, especially slowed processing speed, and indicate that white matter integrity in schizophrenia covaries with individual differences in the severity of processing speed impairment.

Several limitations of our investigation merit discussion before considering the potential implications of our findings. First, the sample sizes, while comparable to most psychiatric neuroimaging investigations, are modest for mediation analysis. For instance, power analysis based on Fitz and MacKinnon (2007) indicates that we were well-powered (i.e. >80\%) to detect large effect sizes for the ' $a$ ' and ' $b$ ' paths of the mediation model for processing speed. However, for the other cognitive variables, which had smaller effects for the ' $b$ ' paths (all Cohen's $d<.30$ ), we would have needed considerably larger sample sizes to reach $80 \%$ power to detect an effect. Thus, while our findings speak to the strength of the relationship between white matter integrity and processing speed impairment in schizophrenia, we can not rule out the possibility that deficits in other cognitive domains are also partially mediated by white matter integrity. Indeed, our ANOCVA analyses revealed that, similar to processing speed, no group differences in white matter were detected after covarying for verbal learning at the standard $\mathrm{p}=.05$ (corrected) threshold. However, in contrast to the ANCOVA covarying for processing speed, significant group differences remained at the more liberal threshold of $\mathrm{p}=.10$ (corrected) after covarying for verbal learning. This suggests that verbal learning also 
accounts for some of the variability in reduced white matter integrity in schizophrenia, but less than processing speed. Larger sample sizes will likely be needed to more accurately determine the extent to which specific cognitive domains are related to white matter pathology in schizophrenia. A Second limitation is that the SCIP, while a very good approximate of overall cognitive functioning, is not a replacement for a comprehensive neuropsychological assessment (Cuesta et al. 2011). It's possible that other cognitive domains, in addition to processing speed, might have been linked to white matter integrity had we used a more extensive battery. However, the fact that a specific relationship between processing speed and white matter was detected with a relatively brief battery is all the more striking as more extensive neuropsychological batteries generally result in greater independence between cognitive domains, not less, and greater power for detecting specific brain-behavior relationships. Additionally, processing speed, as measured with commonly used neuropsychological tests, taps a wide range of cognitive abilities raising questions about which specific cognitive operations contributing to neuropsychological tests of processing speed are related to white matter integrity. Finer-grained measures capable of parsing the specific cognitive operations involved in processing speed will be required to address this limitation. Finally, our decision to focus on overall white matter integrity derived from voxels identified in the between groups analysis might have limited our ability to detect more spatially circumscribed associations between cognition and white matter. A voxel-wise approach is more sensitive in this case; however, there is considerably debate in the neuroimaging field about the validity of voxel-wise brain-behavior correlation analyses, with some investigators arguing that exploratory, whole-brain voxel-wise correlation analyses yield inflated estimates of effect size when not tested in an independent data set (e.g. Vul and Pashler, 2012). Thus, while our mediation analysis is methodological sound as it focused on mean FA extracted from regions identified a-priori in the between groups analysis, it might be insensitive to detecting white matter-cognition associations that are spatially circumscribed, and the voxel-wise correlation analysis examining the relationship between white matter integrity and cognition in schizophrenia should be interpreted cautiously until replicated.

Consistent with many prior studies, we found reductions in white matter integrity in schizophrenia that included many tracts often implicated in the disorder, including the corpus callosum, cingulum, and anterior corona radiata (Fitzsimmons et al. 2013; Melonakos et al. 2011). However, the findings across studies are remarkably heterogeneous with some investigations reporting widespread decreases in white matter integrity and others reporting circumscribed reductions or, in the case of a small minority of studies, no changes at all (e.g. Nenadic et al. 2011). By linking the severity of white matter pathology to individual differences in processing speed impairment, our results provide a potential explanation for the variable findings. The current results also have implications for dimensional approaches to parsing the heterogeneity of schizophrenia (Cuthbert and Insel, 2013). Specifically, the fact that we did not find any effect of schizophrenia diagnosis on white matter integrity after covarying for processing speed indicates that the changes in white matter associated with the categorical illness variable were accounted for by variability in a specific dimension of the illness. This was not the case for several other cognitive domains prominently affected in schizophrenia indicating that the association 
between processing speed and white matter integrity was unlikely a statistical artifact related to co-linearity (i.e. strong association between processing speed impairment and schizophrenia). Specifically, widespread reductions in the integrity of the corpus callosum and prefrontal cortex white matter remained after covarying for working memory, executive functions, and verbal memory, all of which were impaired in schizophrenia. Our results make a compelling case that white matter pathology in schizophrenia is related to cognitive impairment and provide further support for dimensional approaches to dissecting the heterogeneity of schizophrenia.

The association between processing speed impairment and reduced white matter integrity in schizophrenia, while correlational in nature, raises the possibility that the two features result from a common etiological mechanism. The high heritability of schizophrenia, along with strong evidence that white matter integrity, inferred from FA, and processing speed are also heritable, implicates a genetic basis (Doherty et al. 2012; Jahanshad et al. 2013; Toulopoulou et al. 2007). This is supported by evidence that the integrity of white matter tracts prominently affected in probands, such as the corpus callosum, is reduced in unaffected relatives of patients (Knochel et al. 2012). Imaging genetic studies have linked allelic variation in a number of putative schizophrenia risk genes, including CNTNAP2, NTRK1, NTRK3, and MIR137, to white matter integrity (Braskie et al. 2012; Braskie et al. 2013; Clemm von et al. 2013; Lett et al. 2013). However, there are also negative findings. For instance, the genome-wide risk gene ZNF804A appears to be unrelated to white matter integrity (Sprooten et al. 2012; Wei et al. 2013). Recently, Voineskos et al. (2012) found that the effect of several risk alleles in oligodendrocyte genes on cognition, including MAG, CNP, and OLIG2, was mediated by white matter integrity, thus establishing a putative pathway linking genetic variation within white matter genes to white matter integrity and, ultimately, cognition (Voineskos et al. 2012).

The current results may also have implications for the prevention and treatment of cognitive impairment in schizophrenia. Both deficient processing speed and reductions in white matter integrity are observed in individuals at high-risk for psychosis, with those who go on to develop a full-blown psychotic disorder demonstrating worse cognition and more white matter pathology than non-converters (Carletti et al. 2012; Karlsgodt et al. 2009). In patients with schizophrenia, chronic patients demonstrate greater white matter changes than first episode patients (Friedman et al. 2008). It is not known if cognitive decline and changes in anatomical brain connectivity move in parallel during the transition to psychosis, or over the course of the illness. However, interventions designed to preserve the integrity of white matter in high-risk populations, or ameliorate white matter deterioration in patients, may ward off cognitive impairment and/or reduce deterioration over the course of the illness. Moreover, evidence that processing speed impairment mediates deficits in other cognitive domains suggests that the benefits of improving processing speed could be widespread (Andersen et al. 2013; Rodriguez-Sanchez et al. 2007). For instance, Anderson et al. (2013) found that deficits in other cognitive domains, including working memory, verbal memory, and executive function were mediated by processing speed. Mounting pre-clinical and clinical evidence supporting myelin dysfunction in schizophrenia implies that drugs that improve or preserve myelin might prove effective in the disorder, as some have suggested (Walterfang et al. 2011). There is also accumulating evidence that cognitive remediation 
improves white matter integrity. Recently, Penades et al. (2013), found that white matter integrity of the genu of the corpus callosum, a region which mediated processing speed impairment in the current investigation, increased to a greater extent in patients receiving cognitive remediation compared to a treatment control group. Although Penades et al (2013) found no effect of cognitive remediation on processing speed, a recent meta-analysis concluded that cognitive remediation does have a modest effect on processing speed (Wykes et al. 2011). However, it is important to note that processing speed impairment was only partially mediated by white matter integrity. Clearly, other factors, including possible grey matter changes and functional abnormalities, also contribute to processing speed dysfunction.

In conclusion, we found that cognitive functioning, particularly processing speed impairment in schizophrenia, is mediated by decreased white matter integrity. Moreover, we did not detect any differences in white matter integrity between healthy subjects and individuals with schizophrenia after accounting for individual differences in processing speed. Importantly, the relationship between white matter and cognitive impairment was most robust for processing speed; deficits in other cognitive domains affected in schizophrenia, including working memory, verbal memory, and overall cognitive impairment, were not mediated by white matter integrity. However, larger sample sizes will be required to determine the extent to which deficits in other cognitive domains are mediated by white matter integrity. The results suggest a common etiological mechanism, possibly genetic, might underlie processing speed impairment and white matter pathology, and further imply that interventions that preserve and/or improve white matter integrity may enhance cognition.

\section{Supplementary Material}

Refer to Web version on PubMed Central for supplementary material.

\section{Acknowledgments}

This research was supported by funding from the Brain and Behavior Research Fund (NARSAD Young Investigator Award awarded to NDW), the Jack Martin, M.D., Research Professorship in Psychopharmacology (held by NDW), the National Institute of Mental Health (K01-MH083052 awarded to JUB), and the Vanderbilt University Psychiatric Genotype/Phenotype Project.

\section{Reference List}

Andersen R, Fagerlund B, Rasmussen H, Ebdrup BH, Aggernaes B, Gade A, Oranje B, Glenthoj B. the influence of impaired processing speed on cognition in first-episode antipsychotic-naive schizophrenic patients. Eur Psychiatry. 2013; 28:332-339. [PubMed: 22999439]

Behrens TEJ, Woolrich MW, Jenkinson M, Johansen-Berg H, Nunes RG, Clare S, Matthews PM, Brady JM, Smith SM. Characterization and propagation of uncertainty in diffusion-weighted MR imaging. Magnetic Resonance in Medicine. 2003; 50:1077-1088. [PubMed: 14587019]

Bendlin BB, Fitzgerald ME, Ries ML, Xu G, Kastman EK, Thiel BW, Rowley HA, Lazar M, Alexander AL, Johnson SC. White matter in aging and cognition: a cross-sectional study of microstructure in adults aged eighteen to eighty-three. Dev Neuropsychol. 2010; 35:257-277. [PubMed: 20446132]

Braskie MN, Jahanshad N, Stein JL, Barysheva M, Johnson K, McMahon KL, de Zubicaray GI, Martin NG, Wright MJ, Ringman JM, Toga AW, Thompson PM. Relationship of a variant in the 
NTRK1 gene to white matter microstructure in young adults. J Neurosci. 2012; 32:5964-5972. [PubMed: 22539856]

Braskie MN, Kohannim O, Jahanshad N, Chiang MC, Barysheva M, Toga AW, Ringman JM, Montgomery GW, McMahon KL, de Zubicaray GI, Martin NG, Wright MJ, Thompson PM. Relation between variants in the neurotrophin receptor gene, NTRK3, and white matter integrity in healthy young adults. Neuroimage. 2013; 82C:146-153.

Carletti F, Woolley JB, Bhattacharyya S, Perez-Iglesias R, Fusar PP, Valmaggia L, Broome MR, Bramon E, Johns L, Giampietro V, Williams SC, Barker GJ, McGuire PK. Alterations in white matter evident before the onset of psychosis. Schizophr Bull. 2012; 38:1170-1179. [PubMed: 22472474]

Clemm von HC, Wigand MC, Kubicki M, Leicht G, Giegling I, Karch S, Hartmann AM, Konte B, Friedl M, Ballinger T, Eckbo R, Bouix S, Jager L, Shenton ME, Rujescu D, Mulert C. CNTNAP2 polymorphisms and structural brain connectivity: A diffusion-tensor imaging study. J Psychiatr Res. 2013

Cuesta MJ, Pino O, Guilera G, Rojo JE, Gomez-Benito J, Purdon SE, Franco M, Martinez-Aran A, Segarra N, Tabares-Seisdedos R, Vieta E, Bernardo M, Crespo-Facorro B, Mesa F, Rejas J. Brief cognitive assessment instruments in schizophrenia and bipolar patients, and healthy control subjects: a comparison study between the Brief Cognitive Assessment Tool for Schizophrenia (BCATS) and the Screen for Cognitive Impairment in Psychiatry (SCIP). Schizophr Res. 2011; 130:137-142. [PubMed: 21652178]

Cuthbert BN, Insel TR. Toward the future of psychiatric diagnosis: the seven pillars of RDoC. BMC Med. 2013; 11:126. [PubMed: 23672542]

Dickinson D, Ragland JD, Gold JM, Gur RC. General and specific cognitive deficits in schizophrenia: Goliath defeats David? Biol Psychiatry. 2008; 64:823-827. [PubMed: 18472089]

Dickinson D, Ramsey ME, Gold JM. Overlooking the obvious: a meta-analytic comparison of digit symbol coding tasks and other cognitive measures in schizophrenia. Arch Gen Psychiatry. 2007; 64:532-542. [PubMed: 17485605]

Doherty JL, O’Donovan MC, Owen MJ. Recent genomic advances in schizophrenia. Clin Genet. 2012; 81:103-109. [PubMed: 21895634]

First, MB., Spitzer, RL., Gibbon, M., Williams, JBW. Structured Clinical Interview for DSM-IV Axis I Disorders, Clinical Version (SCID-CV). American Psychiatric Press Inc; Washington, D.C: 1996.

Fitzsimmons J, Kubicki M, Shenton ME. Review of functional and anatomical brain connectivity findings in schizophrenia. Curr Opin Psychiatry. 2013; 26:172-187. [PubMed: 23324948]

Friedman JI, Tang C, Carpenter D, Buchsbaum M, Schmeidler J, Flanagan L, Golembo S, Kanellopoulou I, Ng J, Hof PR, Harvey PD, Tsopelas ND, Stewart D, Davis KL. Diffusion tensor imaging findings in first-episode and chronic schizophrenia patients. Am J Psychiatry. 2008; 165:1024-1032. [PubMed: 18558643]

Fritz MS, MacKinnon DP. Required sample size to detect the mediated effect. Psychol Sci. 2007; 18:233-239. [PubMed: 17444920]

Fusar-Poli P, Deste G, Smieskova R, Barlati S, Yung AR, Howes O, Stieglitz RD, Vita A, McGuire P, Borgwardt S. Cognitive functioning in prodromal psychosis: a meta-analysis. Arch Gen Psychiatry. 2012; 69:562-571. [PubMed: 22664547]

Gardner DM, Murphy AL, O’Donnell H, Centorrino F, Baldessarini RJ. International consensus study of antipsychotic dosing. Am J Psychiatry. 2010; 167:686-693. [PubMed: 20360319]

Hayes AF. Beyond Baron and Kenny: statistical mediation analysis in the new millennium. Communication Mongraphs. 2009; 76:408-420.

Heinrichs RW, Zakzanis KK. Neurocognitive deficit in schizophrenia: a quantitative review of the evidence. Neuropsychology. 1998; 12:426-445. [PubMed: 9673998]

Jahanshad N, Kochunov PV, Sprooten E, Mandl RC, Nichols TE, Almasy L, Blangero J, Brouwer RM, Curran JE, de Zubicaray GI, Duggirala R, Fox PT, Hong LE, Landman BA, Martin NG, McMahon KL, Medland SE, Mitchell BD, Olvera RL, Peterson CP, Starr JM, Sussmann JE, Toga AW, Wardlaw JM, Wright MJ, Hulshoff Pol HE, Bastin ME, McIntosh AM, Deary IJ, Thompson PM, Glahn DC. Multi-site genetic analysis of diffusion images and voxelwise heritability analysis: A pilot project of the ENIGMA-DTI working group. Neuroimage. 2013; 81C:455-469. 
Karlsgodt KH, Niendam TA, Bearden CE, Cannon TD. White matter integrity and prediction of social and role functioning in subjects at ultra-high risk for psychosis. Biol Psychiatry. 2009; 66:562569. [PubMed: 19423081]

Karlsgodt KH, van Erp TGM, Poldrack RA, Bearden CE, Nuechterlein KH, Cannon TD. Diffusion Tensor Imaging of the Superior Longitudinal Fasciculus and Working Memory in Recent-Onset Schizophrenia. Biological Psychiatry. 2008; 63:512-518. [PubMed: 17720147]

Kerchner GA, Racine CA, Hale S, Wilheim R, Laluz V, Miller BL, Kramer JH. Cognitive processing speed in older adults: relationship with white matter integrity. PLoS One. 2012; 7:e50425. [PubMed: 23185621]

Knochel C, Oertel-Knochel V, Schonmeyer R, Rotarska-Jagiela A, van dV, Prvulovic V, Haenschel D, Uhlhaas C, Pantel P, Hampel JH, Linden DE. Interhemispheric hypoconnectivity in schizophrenia: fiber integrity and volume differences of the corpus callosum in patients and unaffected relatives. Neuroimage. 2012; 59:926-934. [PubMed: 21964509]

Koch K, Wagner G, Schachtzabel C, Schultz CC, Gullmar D, Reichenbach JR, Sauer H, Schlosser RG. Age-dependent visuomotor performance and white matter structure: a DTI study. Brain Struct Funct. 2012

Lett TA, Chakavarty MM, Felsky D, Brandl EJ, Tiwari AK, Goncalves VF, Rajji TK, Daskalakis ZJ, Meltzer HY, Lieberman JA, Lerch JP, Mulsant BH, Kennedy JL, Voineskos AN. The genome-wide supported microRNA-137 variant predicts phenotypic heterogeneity within schizophrenia. Mol Psychiatry. 2013; 18:443-450. [PubMed: 23459466]

Levitt JJ, Alvarado JL, Nestor PG, Rosow L, Pelavin PE, McCarley RW, Kubicki M, Shenton ME. Fractional anisotropy and radial diffusivity: diffusion measures of white matter abnormalities in the anterior limb of the internal capsule in schizophrenia. Schizophr Res. 2012; 136:55-62. [PubMed: 22019073]

MacKinnon DP, Lockwood CM, Hoffman JM, West SG, Sheets V. A comparison of methods to test mediation and other intervening variable effects. Psychol Methods. 2002; 7:83-104. [PubMed: 11928892]

Marenco S, Stein JL, Savostyanova AA, Sambataro F, Tan HY, Goldman AL, Verchinski BA, Barnett AS, Dickinson D, Apud JA, Callicott JH, Meyer-Lindenberg A, Weinberger DR. Investigation of anatomical thalamo-cortical connectivity and FMRI activation in schizophrenia. Neuropsychopharmacology. 2012; 37:499-507. [PubMed: 21956440]

Melonakos ED, Shenton ME, Rathi Y, Terry DP, Bouix S, Kubicki M. Voxel-based morphometry (VBM) studies in schizophrenia-can white matter changes be reliably detected with VBM? Psychiatry Res. 2011; 193:65-70. [PubMed: 21684124]

Nenadic I, Wagner G, Gullmar D, Schachtzabel C, von CK, Kohler S, Koch K, Roebel M, Schultz CC, Reichenbach JR, Sauer H, Schlosser RG. ADC changes in schizophrenia: a diffusion-weighted imaging study. Eur Arch Psychiatry Clin Neurosci. 2011; 261:213-216. [PubMed: 20872228]

Nestor PG, Kubicki M, Gurrera RJ, Niznikiewicz M, Frumin M, McCarley RW, Shenton ME. Neuropsychological correlates of diffusion tensor imaging in schizophrenia. Neuropsychology. 2004; 18:629-637. [PubMed: 15506830]

Nestor PG, Kubicki M, Nakamura M, Niznikiewicz M, McCarley RW, Shenton ME. Comparing prefrontal gray and white matter contributions to intelligence and decision making in schizophrenia and healthy controls. Neuropsychology. 2010; 24:121-129. [PubMed: 20063953]

Nuechterlein KH, Subotnik KL, Green MF, Ventura J, Asarnow RF, Gitlin MJ, Yee CM, GretchenDoorly D, Mintz J. Neurocognitive predictors of work outcome in recent-onset schizophrenia. Schizophr Bull. 2011; 37(Suppl 2):S33-S40. [PubMed: 21860045]

Ojeda N, Pena J, Sanchez P, Elizagarate E, Ezcurra J. Processing speed mediates the relationship between verbal memory, verbal fluency, and functional outcome in chronic schizophrenia. Schizophr Res. 2008; 101:225-233. [PubMed: 18261885]

Penades R, Pujol N, Catalan R, Massana G, Rametti G, Garcia-Rizo C, Bargallo N, Gasto C, Bernardo $\mathrm{M}$, Junque $\mathrm{C}$. Brain effects of cognitive remediation therapy in schizophrenia: a structural and functional neuroimaging study. Biol Psychiatry. 2013; 73:1015-1023. [PubMed: 23452665] 
Penke L, Maniega SM, Bastin ME, Valdes Hernandez MC, Murray C, Royle NA, Starr JM, Wardlaw JM, Deary IJ. Brain white matter tract integrity as a neural foundation for general intelligence. Mol Psychiatry. 2012; 17:1026-1030. [PubMed: 22614288]

Penke L, Munoz MS, Murray C, Gow AJ, Hernandez MC, Clayden JD, Starr JM, Wardlaw JM, Bastin ME, Deary IJ. A general factor of brain white matter integrity predicts information processing speed in healthy older people. J Neurosci. 2010; 30:7569-7574. [PubMed: 20519531]

Perez-Iglesias R, Tordesillas-Gutierrez D, McGuire PK, Barker GJ, Roiz-Santianez R, Mata I, de Lucas EM, Rodriguez-Sanchez JM, Ayesa-Arriola R, Vazquez-Barquero JL, Crespo-Facorro B. White matter integrity and cognitive impairment in first-episode psychosis. Am J Psychiatry. 2010; 167:451-458. [PubMed: 20160006]

Preacher KJ, Hayes AF. SPSS and SAS procedures for estimating indirect effects in simple mediation models. Behav Res Methods Instrum Comput. 2004; 36:717-731. [PubMed: 15641418]

Purdon, SE. The Screen for Cognitive Impairment in Psychiatry (SCIP): Administration Manual and Normative Data. PNL Inc; Edmonton, Alberta: 2005.

Qiu A, Zhong J, Graham S, Chia MY, Sim K. Combined analyses of thalamic volume, shape and white matter integrity in first-episode schizophrenia. Neuroimage. 2009; 47:1163-1171. [PubMed: 19375511]

Rodriguez-Sanchez JM, Crespo-Facorro B, Gonzalez-Blanch C, Perez-Iglesias R, Vazquez-Barquero JL. Cognitive dysfunction in first-episode psychosis: the processing speed hypothesis. Br J Psychiatry Suppl. 2007; 51:s107-s110. [PubMed: 18055925]

Salami A, Eriksson J, Nilsson LGr, Nyberg L. Age-related white matter microstructural differences partly mediate age-related decline in processing speed but not cognition. Biochimica et Biophysica Acta (BBA) - Molecular Basis of Disease. 2012; 1822:408-415. [PubMed: 21930202]

Sanchez P, Ojeda N, Pena J, Elizagarate E, Yoller AB, Gutierrez M, Ezcurra J. Predictors of longitudinal changes in schizophrenia: the role of processing speed. J Clin Psychiatry. 2009; 70:888-896. [PubMed: 19422757]

Sasson E, Doniger GM, Pasternak O, Tarrasch R, Assaf Y. White matter correlates of cognitive domains in normal aging with diffusion tensor imaging. Front Neurosci. 2013; 7:32. [PubMed: 23493587]

Smith SM. Fast robust automated brain extraction. Hum Brain Mapp. 2002; 17:143-155. [PubMed: 12391568]

Smith SM, Jenkinson M, Johansen-Berg H, Rueckert D, Nichols TE, Mackay CE, Watkins KE, Ciccarelli O, Cader MZ, Matthews PM, Behrens TE. Tract-based spatial statistics: voxelwise analysis of multi-subject diffusion data. Neuroimage. 2006; 31:1487-1505. [PubMed: 16624579]

Smith SM, Jenkinson M, Woolrich MW, Beckmann CF, Behrens TE, Johansen-Berg H, Bannister PR, De LM, Drobnjak I, Flitney DE, Niazy RK, Saunders J, Vickers J, Zhang Y, De SN, Brady JM, Matthews PM. Advances in functional and structural MR image analysis and implementation as FSL. Neuroimage. 2004; 23(Suppl 1):S208-S219. [PubMed: 15501092]

Smith SM, Nichols TE. Threshold-free cluster enhancement: Addressing problems of smoothing, threshold dependence and localisation in cluster inference. NeuroImage. 2009; 44:83-98. [PubMed: 18501637]

Sprooten E, McIntosh AM, Lawrie SM, Hall J, Sussmann JE, Dahmen N, Konrad A, Bastin ME, Winterer G. An investigation of a genomewide supported psychosis variant in ZNF804A and white matter integrity in the human brain. Magn Reson Imaging. 2012; 30:1373-1380. [PubMed: 22840435]

Szeszko PR, Robinson DG, Ashtari M, Vogel J, Betensky J, Sevy S, Ardekani BA, Lencz T, Malhotra AK, McCormack J, Miller R, Lim KO, Gunduz-Bruce H, Kane JM, Bilder RM. Clinical and Neuropsychological Correlates of White Matter Abnormalities in Recent Onset Schizophrenia. Neuropsychopharmacology. 2007; 33:976-984. [PubMed: 17581532]

Takei K, Yamasue H, Abe O, Yamada H, Inoue H, Suga M, Muroi M, Sasaki H, Aoki S, Kasai K. Structural disruption of the dorsal cingulum bundle is associated with impaired Stroop performance in patients with schizophrenia. Schizophr Res. 2009; 114:119-127. [PubMed: 19505800] 
Toulopoulou T, Picchioni M, Rijsdijk F, Hua-Hall M, Ettinger U, Sham P, Murray R. Substantial genetic overlap between neurocognition and schizophrenia: genetic modeling in twin samples. Arch Gen Psychiatry. 2007; 64:1348-1355. [PubMed: 18056542]

Tuch DS, Salat DH, Wisco JJ, Zaleta AK, Hevelone ND, Rosas HD. Choice reaction time performance correlates with diffusion anisotropy in white matter pathways supporting visuospatial attention. Proc Natl Acad Sci U S A. 2005; 102:12212-12217. [PubMed: 16103359]

Turken A, Whitfield-Gabrieli S, Bammer R, Baldo JV, Dronkers NF, Gabrieli JD. Cognitive processing speed and the structure of white matter pathways: convergent evidence from normal variation and lesion studies. Neuroimage. 2008; 42:1032-1044. [PubMed: 18602840]

Voineskos AN, Felsky D, Kovacevic N, Tiwari AK, Zai C, Chakravarty MM, Lobaugh NJ, Shenton ME, Rajji TK, Miranda D, Pollock BG, Mulsant BH, McIntosh AR, Kennedy JL. Oligodendrocyte Genes, White Matter Tract Integrity, and Cognition in Schizophrenia. Cereb Cortex. 2012

Vul E, Pashler H. Voodoo and circularity errors. Neuroimage. 2012; 62:945-948. [PubMed: 22270348]

Walterfang M, Velakoulis D, Whitford TJ, Pantelis C. Understanding aberrant white matter development in schizophrenia: an avenue for therapy? Expert Rev Neurother. 2011; 11:971-987. [PubMed: 21721915]

Wechsler, D. Wechsler Adult Intelligence Scale. 3. The Psychological Corporation; 1997.

Wechsler, D. Wechsler Test of Adult Reading. Pearson Education; 2001.

Wei Q, Kang Z, Diao F, Guidon A, Wu X, Zheng L, Li L, Guo X, Hu M, Zhang J, Liu C, Zhao J. No association of ZNF804A rs1344706 with white matter integrity in schizophrenia: a tract-based spatial statistics study. Neurosci Lett. 2013; 532:64-69. [PubMed: 23147122]

Wykes T, Huddy V, Cellard C, McGurk SR, Czobor P. A meta-analysis of cognitive remediation for schizophrenia: methodology and effect sizes. Am J Psychiatry. 2011; 168:472-485. [PubMed: 21406461]

Yao L, Lui S, Liao Y, Du MY, Hu N, Thomas JA, Gong QY. White matter deficits in first episode schizophrenia: An activation likelihood estimation meta-analysis. Prog Neuropsychopharmacol Biol Psychiatry. 2013 


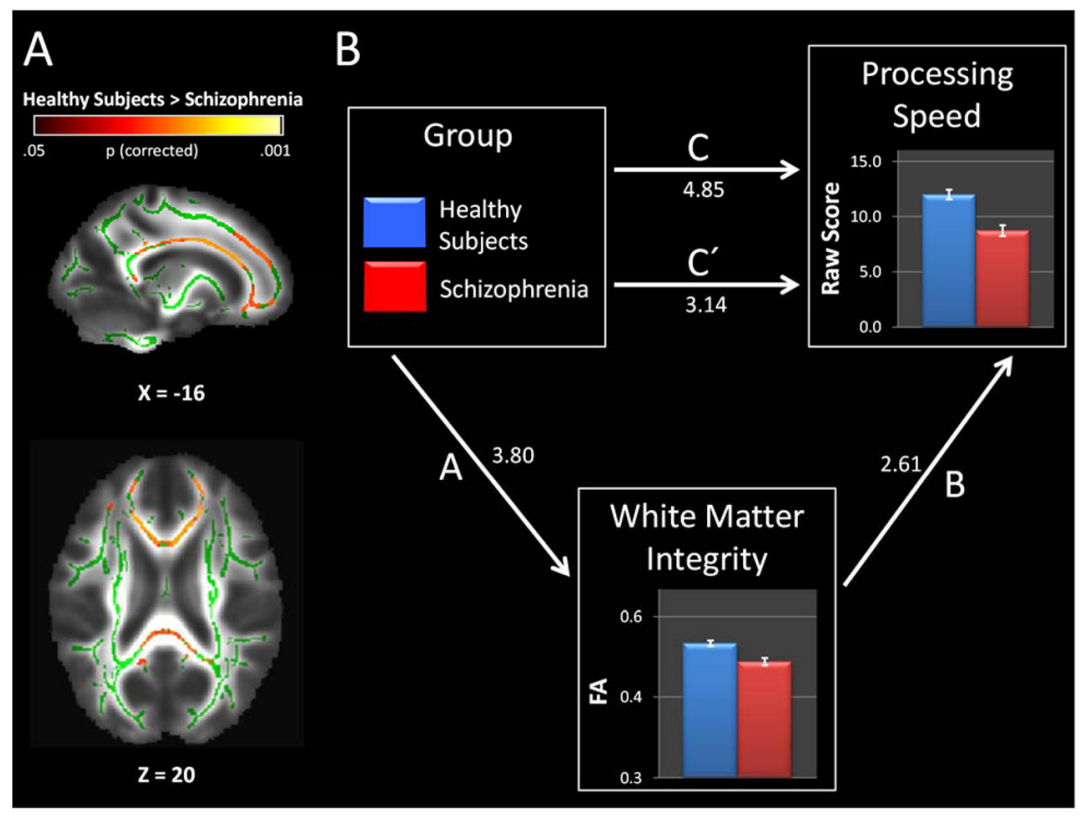

Figure 1.

Reduced white matter integrity and processing speed impairment in schizophrenia. Panel A: White matter integrity, inferred from fractional anisotropy (FA), was reduced in several brain areas in schizophrenia including the corpus callosum, superior frontal gyrus white matter, and precuneus white matter. Areas demonstrating reduced FA in schizophrenia (i.e. warm colors) are overlaid on the skeletonized FA map (i.e. green). Panel B: Processing speed impairment in schizophrenia was partially mediated by mean FA from the regions that demonstrated lower FA in patients compared to healthy subjects. Standardized coefficients, all of which were significant ( $\mathrm{p}<.05)$, are shown for each pathway. Sobel test confirmed that the strength of the association between the independent variable, group, and dependent variable, processing speed, was reduced after accounting for white matter integrity (path $\mathrm{C}$ vs. $C^{\prime}: \beta=.48$, bootstrap Confidence Interval $\left.=.18-.92, p<.05\right)$. 


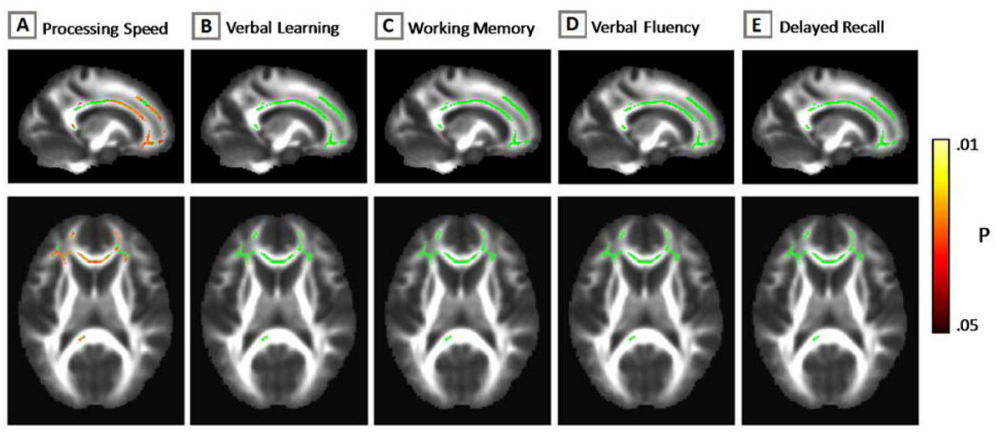

Figure 2.

Correlations between white matter integrity, inferred from fractional anisotropy (FA), and cognition in schizophrenia. Processing speed correlated with FA in corpus callosum and superior frontal gyrus (Panel A). White matter integrity was not correlated with other cognitive domains (Panels B-E). 
A White Matter Integrity (FA): Control > Schizophrenia

$\mathrm{p}$ (corrected) $<.05$
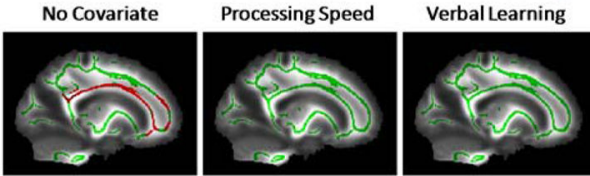

Working Memory
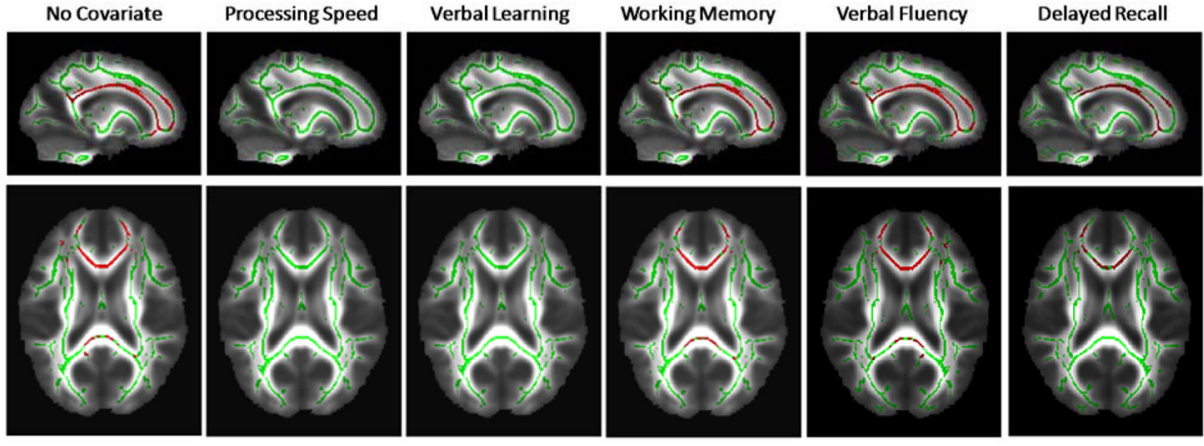

B White Matter Integrity (FA): Control > Schizophrenia

$\mathrm{p}($ corrected $)<.10$
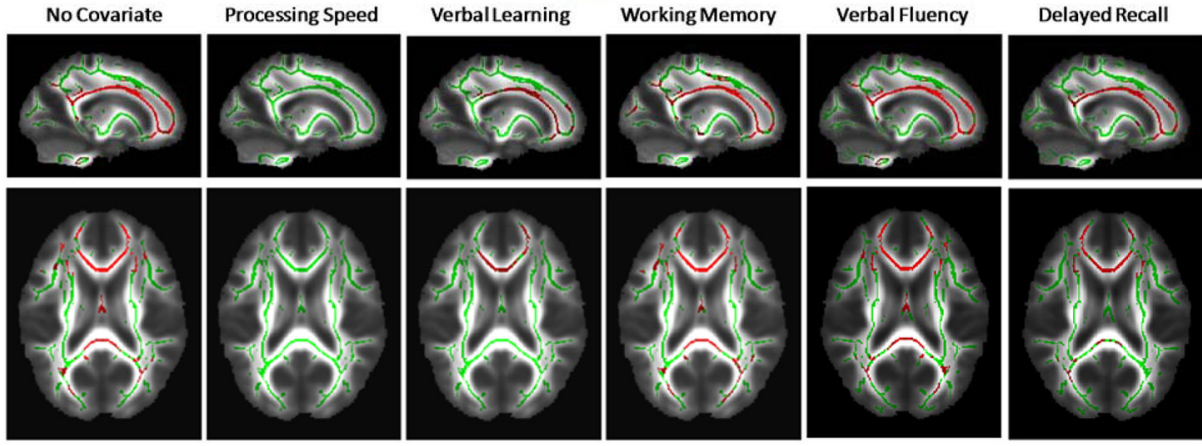

Figure 3.

White matter integrity reductions in schizophrenia with and without covarying for cognition. Without covarying for cognitive impairment, white matter integrity, inferred from fractional anisotropy (FA), was reduced in multiple brain areas in schizophrenia at both conservative ( $\mathrm{p}<.05$; Panel A) and liberal statistical thresholds ( $\mathrm{p}<.10$; Panel B). Similar results were observed after covarying for working memory, verbal fluency, delayed recall, and, to a lesser extent, verbal learning. In contrast, no group differences were observed after covarying for processing speed at either the conservative or liberal statistical thresholds. Statistical parametric maps (i.e. warm colors) are overlaid on the skeletonized FA map (i.e. green). 

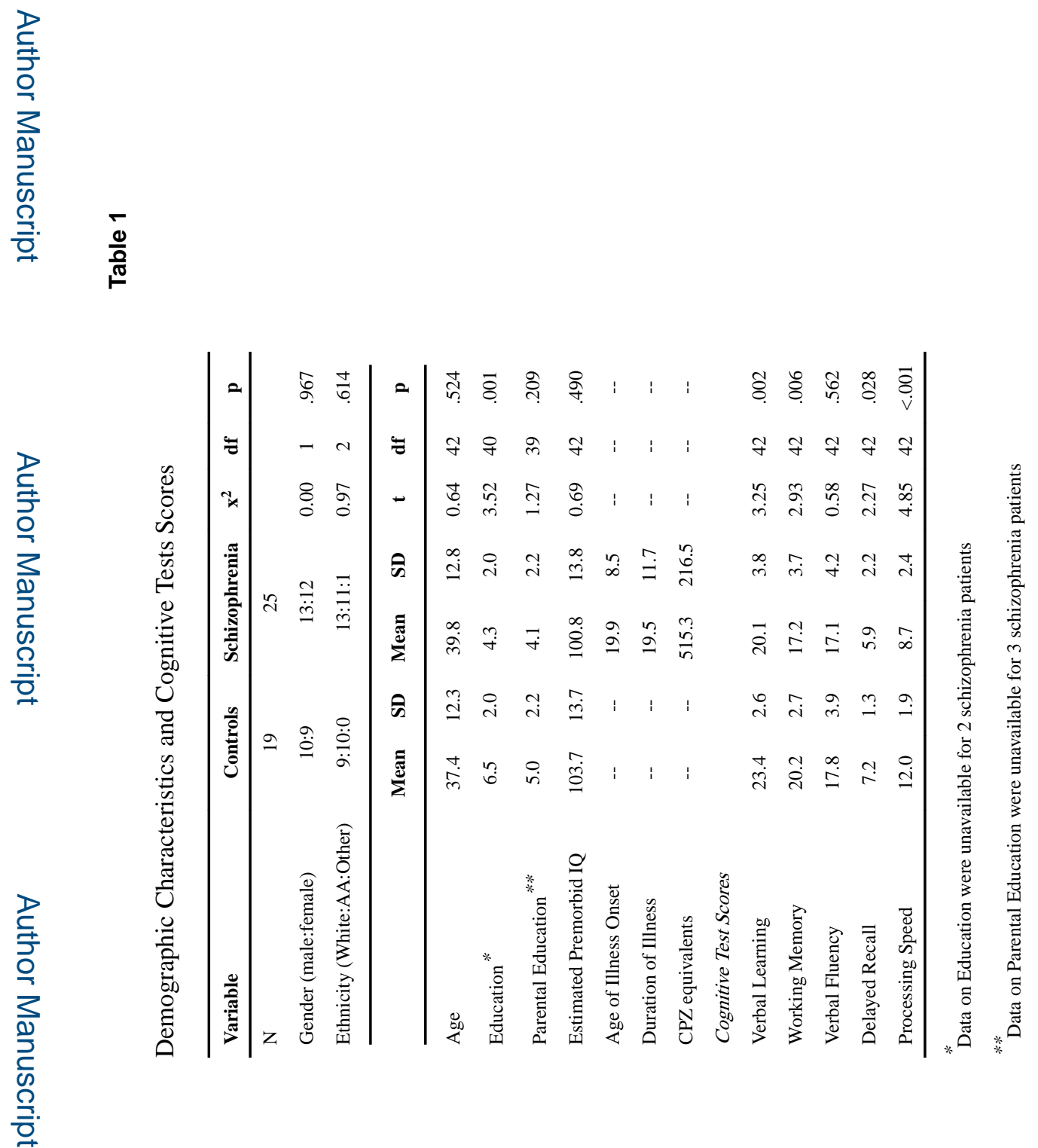

Psychol Med. Author manuscript; available in PMC 2017 February 08. 